\title{
ENTROPIES, PARTITIONINGS AND HEART RATE VARIABILITY
}

\author{
Milan Palus $^{1^{*}}$ and Jan J. Zebrowski ${ }^{2}$ \\ ${ }^{1}$ Institute of Computer Science, Academy of Sciences of the Czech Republic, Prague, Czech Republic \\ ${ }^{2}$ Institute of Physics, Warsaw University of Technology, Warszawa, Poland
}

Received April 20, 2009; accepted April 30, 2009

\begin{abstract}
Several definitions of static entropies and coarse-grained entropy rates (CER's) were used to analyze 24-hour RR interval sequences of three sex, age and disease matched control pairs. The matching was done with the assessment of the risk of cardiac arrest in mind. Details of estimating probability densities (histograms), used to compute the static entropies, have been found crucial to obtain the proper discrimination between the members of control pairs. The effect of signal variance on the entropy estimation is discussed. In a preliminary result, CER's have been found to yield additional information which may be helpful in discriminating health and pathology.
\end{abstract}

\section{Key words: Coarse-grained entropy rate; HR variability; Entropy}

\section{INTRODUCTION}

This study was inspired by the basic question, which is often asked by all time-series analysts dealing with physiological data, namely, which property of the data discriminates health from pathology and how this property can be described and measured, so that a reliable diagnostic tool is obtained. We investigated data characterizing heart-rate variability - interbeat intervals, called also the RR intervals. We used RR interval series, forming 3 sex, age and disease matched control pairs. These data pairs were chosen to test whether any given technique of nonlinear dynamical analysis is able to correctly distinguish between a person at risk of a cardiac arrest (CA) and his/her control. Below the term "health, healthy" will mean control, while the term "pathology" will signify a person with a high risk of the cardiac arrest. Note, that a sex, age and disease matched control medically resembles the target person in as many respects as possible, except for the single feature investigated - in this case the risk of the cardiac arrest.

It has been questioned, whether the health-pathology differences are encoded in the dynamics of the RR interval series, characterized by their entropy rates, or in the geometry of "phase portraits", obtained by timelagging the series and characterized by 2- and 3dimensional Shannon entropies, or even in the static

\footnotetext{
*E-mail:mp@cs.cas.cz
}

marginal distributions (histograms) of the series, characterized by 1-dimensional Shannon entropies, and eventually in the higher-order properties of the latter. The higher-order properties of the probability distributions are characterized by Renyi entropies and also by complexity measures called pattern entropies as introduced by Zebrowski et al. (1994, 1999, 2000).

We present a partial answer to this question focusing on practical aspects of the estimations of entropies and entropy-related quantities from experimental data. We found that the results were much more infuenced by a particular method of estimating the entropies (more precisely by a particular method of computing the histograms as estimates of probability distributions used to calculate the entropies themselves) than by the choice of a particular entropy measure. All the static entropies considered below brought consistent results when the same histogram estimation method was used. The number of data series processed was unfortunately not sufficient to decide which of the estimation methods is the best one for the medical problem from which the data studied here were taken.

Dynamic entropies - the entropy rates, estimated by yet another approach, showed to be consistent in some features related to the discrimination between health and pathology. The concept of coarse-grained entropy rates 
has been introduced in (Palus, 1996), more recent development is described in (Palus \& Vejmelka, 2007). A useful review of methods for estimating entropies and information can be found in (Hlavackova, Schindler et al., 2007).

\section{DATA}

Holter ECG 24-hour tapes of both healthy individuals and of cases of heart disease with the highest risk of sudden death were analyzed by commercial software (Del Mar Avionics 563 Strata Scan) at the National Institute of Cardiology in Warszawa, Poland. The data were sampled at $128 \mathrm{~Hz}$ and the time distance between consecutive $\mathrm{R}$ peaks (the RR intervals) were extracted.

No special filtering was used, but RR intervals larger than $2500 \mathrm{~ms}$ were treated as artifacts and ignored. Each of the studied 24-hour sequences of RR intervals was 80,000 to 125,000 data points long and began at about 8 o'clock in the morning. The data were used to create medically matched control pairs.
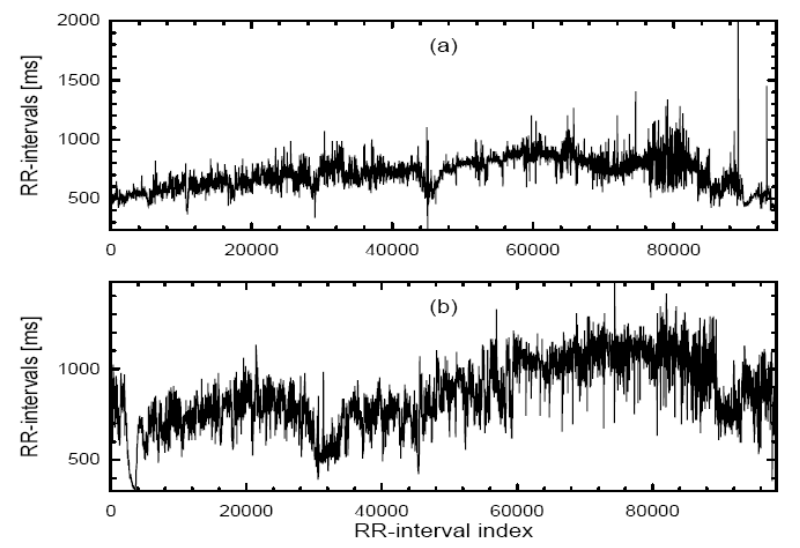

Figure 1. Plot of 24-hour RR-interval series dp1a (a) - the 27 year old male CA patient and b) his control dplb.

We studied 3 sex, age and disease matched control pairs available: all target members of the pairs had had at least a single event of cardiac arrest (CA) in their history. No arrhythmia filters were used. It should be stressed here that the method used to find the appropriate control pair to a given target patient was based on time honoured medical procedures alone. This means that the choice was based only on general medical (clinical) knowledge. Neither nonlinear methods were used to form the sex, age and disease matched pairs, nor time domain and frequency domain measures of ECG were applied, and the RR intervals as functions of the time were not inspected. The members of pair 1 and pair 3 who had undergone CA were labeled "a", their controls were labeled " $b$ ", while the labels of pair 2 were reversed. This was done in order to make the comparison of nonlinear techniques like a blind test. In pair 1, the RR interval series labelled dp1a (Figure 1a) was taken from a 27 year old male who had had CA twice and now wears an implanted defibrillator (which, however, did not activate itself during the Holter recording analysed). All known methods of the ECG analysis in the time and frequency domains, as well as the biopsy of the heart muscle and biochemical analysis, all pronounced him to be in exemplary health. However, pattern entropy (Zebrowski et al., 1994) was found to be at an elevated level which distinguished him well from his control pair dp1b (Figure 1b).

In pair 2, the RR interval series labelled dp2b (Figure 2b) was taken from a 55 year old male who had undergone a myocardial infarction some time ago, complicated by a postinfarction anneurism. He died several months after having been included in the 15 control pairs analysed by pattern entropy and was pronounced at a high risk of CA on the basis of the behavior of this complexity measure. The control for this CA patient is depicted in Figure 2a.
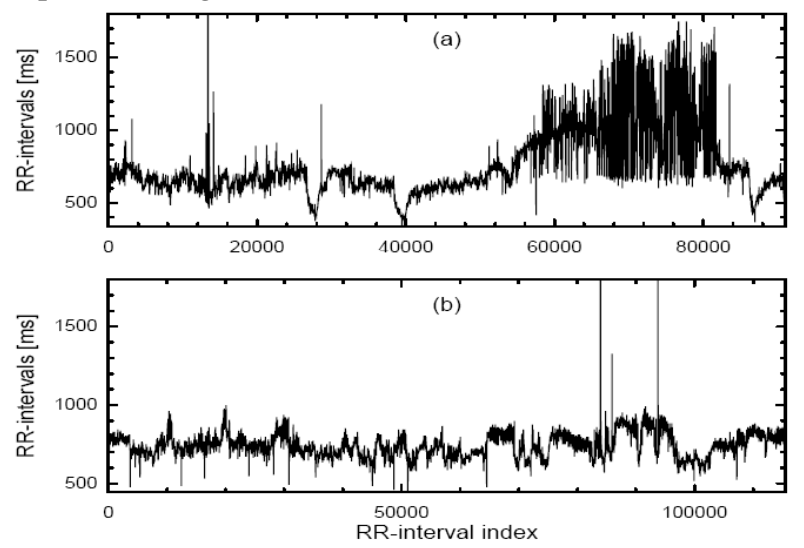

Figure 2. Plot of 24-hour RR-interval series dp2a - the control for $\mathrm{dp} 2 \mathrm{~b}$; b) $\mathrm{dp} 2 \mathrm{~b}-55$ year old male CA patient.

Pair 3 is composed of two recordings of the same person - a 67 year old male also some time after a myocardial infarction. The recording of $\mathrm{dp} 3 \mathrm{a}$ (Figure3a) contains data taken prior and during a CA (when a ventricular fibrillation occurred), subsequent reanimation and ends 1 hour after the CA event. The series dp3b (Figure 3b) was recorded 9 months afterwards and shows the results of medication. Thus, this person became his own sex, age and disease matched control pair.

\section{ENTROPIES AND ENTROPY RATES}

In this section basic definitions of entropies and entropy rates are briefly reviewed. More details can be found in Refs. (Cover \& Thomas, 1991), (Fraser, 1989), (Sinai, 1976), (Petersen, 1983), (Palus, 1993) and references therein. The concept of coarse-grained entropy rates has been introduced in (Palus, 1996), more recent development is described in (Palus \& Vejmelka, 2007). A useful review of methods for estimating entropies and information can be found in Hlavackova, Schindler et al., (2007). 
Let $X$ be a discrete random variable with a set of values $\Xi$ and probability mass function:

$$
p(x)=\operatorname{Pr}\{X=x\}, x \in \Xi
$$

We denote the probability mass function by $p(x)$, rather than $p_{X}(x)$, for convenience.

The Shannon entropy $H(X)$ of a discrete random variable $\mathrm{X}$ is defined by

$$
H(X)=-\sum_{x \in \Xi} p(x) \log p(x)
$$

For $\mathrm{n}$ variables $X_{1}, \ldots, X_{n}$ with the joint distribution $p\left(x_{1}\right.$; $\ldots ; x_{n}$ ) the joint (n-dimensional) Shannon entropy is defined as

$$
H\left(X_{1}, \ldots, X_{n}\right)=-\sum_{x_{1} \in \Xi_{1}} \ldots \sum_{x_{n} \in \Xi_{n}} p\left(x_{1}, \ldots, x_{n}\right) \log p\left(x_{1}, \ldots, x_{n}\right) .
$$

The marginal redundancy $\varrho\left(X_{1} ; \ldots ; X_{n-1} ; X_{n}\right)$ is an information-theoretic measure which quantifies average amount of information about the variable $X_{n}$, contained in the variables $X_{1} ; \ldots ; X_{n-1}$, and is defined as

$$
\varrho\left(X_{1}, \ldots, X_{n-1} ; X_{n}\right)=H\left(X_{1}, \ldots, X_{n-1}\right)+H\left(X_{n}\right)-H\left(X_{1}, \ldots, X_{n}\right) .
$$

In the case of two variables $X_{1} ; X_{2}$, the redundancy $\varrho(X 1 ; X 2)$ is also known as mutual information $I\left(X_{1} ; X_{2}\right)$. Now, let $\left\{X_{i}\right\}$ be a stochastic process, i.e., an indexed sequence of random variables, characterized by the joint probability mass function $p\left(\mathrm{x}_{1} ; \ldots ; \mathrm{x}_{\mathrm{n}}\right)=\operatorname{Pr}\left\{\left(\mathrm{X}_{1} ; \ldots ; \mathrm{X}_{\mathrm{n}}\right)\right.$ $\left.=\left(\mathrm{x}_{1} ; \ldots ; \mathrm{x}_{\mathrm{n}}\right)\right\},\left(\mathrm{x}_{1} ; \ldots ; \mathrm{x}_{\mathrm{n}}\right) \in \Xi_{1} \times \ldots \times \Xi_{n}$. The entropy rate of $\left\{X_{i}\right\}$ is defined as

$$
h=\lim _{n \rightarrow \infty} \frac{1}{n} H\left(X_{1}, \ldots, X_{n}\right),
$$

and can be expressed using the marginal redundancy $\varrho\left(X_{1} ; \ldots ; X_{n-1} ; X_{n}\right)$ as

$$
h=\lim _{n \rightarrow \infty}\left[H\left(X_{n}\right)-\varrho\left(X_{1}, \ldots, X_{n-1} ; X_{n}\right)\right] .
$$

In practical applications a time series $\{y(t)\}$ is considered as a realization of a stationary and ergodic stochastic process $\{Y(t)\}$. Due to ergodicity the entropies and redundancies can be estimated using time averages instead of ensemble averages, and, the variables $X_{i}$ are substituted as

$$
X_{i}=y(t+(i-1) \tau)
$$

Due to stationarity, entropies

$$
H^{n}(\tau) \equiv H(y(t), y(t+\tau), \ldots, y(t+(n-1) \tau))
$$

and marginal redundancies

$$
\varrho^{n}(\tau) \equiv \varrho(y(t), y(t+\tau), \ldots, y(t+(n-2) \tau) ; y(t+(n-1) \tau))
$$

are functions of $n$ and $\tau$, independent of $t$. Then, the entropy rate of $\{y(t)\}$ can be written as

$$
h_{\tau}=\varrho^{n}(0)-\lim _{n \rightarrow \infty} \varrho^{n}(\tau)
$$

For the entropy rate $h_{1}$ per a time unit the following equation holds (Sinai, 1976)

$$
h_{\tau}=|\tau| h_{1}
$$

The possibility to compute the entropy rates from data is limited to a few exceptional cases: for stochastic processes it is possible, e.g., for finite-state Markov chains (Cover and Thomas, 1991). If a time series was generated by a dynamical system, its entropy rate, known as Kolmogorov-Sinai entropy (KSE), can, in principle, be estimated from the asymptotic dependence of the marginal redundancy on time delay (Fraser, 1989):

$$
\lim _{n \rightarrow \infty} \varrho^{n}(\tau)=H^{1}-|\tau| h .
$$

It was shown (Fraser, 1989), (Palus, 1993), (Palus, 1996) that the asymptotic behavior $\varrho^{n}(\tau) \approx H^{l}-|\tau| h$ could be observed in $\varrho^{n}(\tau)$ estimated from time series, however, the data requirements (series length, precision, negligible noise) are not realistic for usual experimental situations such as those in the analysis of physiological time series.
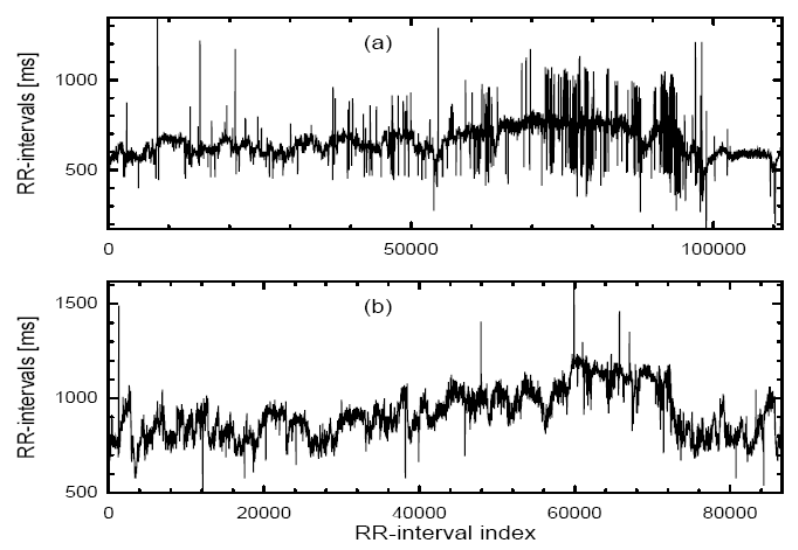

Figure 3. Plot of 24 hour RR-interval series dp3a (a) - the 67 year old male CA patient and b) his control dp3b - the same person after 9 months of medication.

Instead of unsuccessful attempts to estimate the exact entropy rates from real data, Palus (1996b) has proposed "coarse-grained entropy rates" (CER's), computed from the (coarse-grained) marginal redundancy or mutual information. The simplest CER $h^{(0)}$ is defined as 


$$
h^{(0)}=\frac{\varrho^{n}\left(\tau_{0}\right)-\varrho^{n}\left(\tau_{1}\right)}{\tau_{1}-\tau_{0}},
$$

where $\tau_{0}$ and $\tau_{1}$ are parameters of the method to be chosen by a user.

For an alternative CER $h^{(l)}$, which has better numerical properties than $h^{(0)}$, one should compute the marginal redundancies $\varrho^{n}(\tau)$ for all analyzed datasets and find such $\tau_{\text {max }}$ that for $\tau^{\prime} \geq \tau_{\max }: \varrho^{n}\left(\tau^{\prime}\right) \approx 0$ for all the datasets. Then a norm of the marginal redundancy is defined

$$
\left\|\varrho^{n}\right\|=\frac{\sum_{\tau=\tau_{0}}^{\tau_{\max }} \varrho^{n}(\tau)}{\tau_{\max }-\tau_{0}} .
$$

Here the lag $\tau_{\max }$ is derived from the data, the lag $\tau_{0}$ is usually set to zero.

Having defined the norm $\left\|\varrho^{n}\right\|$, the difference $\varrho^{n}\left(\tau_{0}\right)$ $\left\|\varrho^{n}\right\|$ can be considered as the alternative definition of the CER. It was found, however, that the definition of the CER, which does not depend on the absolute values of $\varrho^{n}(\tau)$, has better numerical properties, namely the estimates are more stable and less influenced by noise. Thus, the CER $\mathrm{h}^{(1)}$ is defined as

$$
h^{(1)}=\frac{\varrho^{n}\left(\tau_{0}\right)-\left\|\varrho^{n}\right\|}{\left\|\varrho^{n}\right\|} .
$$

Some aspects of the estimation of the CER's will be discussed in the next section, for more details and description of properties of the CER's see Ref. (Palus, 1996).

The Shannon entropy $H(X) \equiv H^{l}$ evaluates properties of the probability function $p(x)$. Higher-order properties of $p(x)$ can be characterized by Renyi entropies. The Renyi entropy $H_{q}(X)$ of order $q$ for a discrete random variable $X$ is defined as

$$
H_{q}(X)=\frac{1}{1-q} \log \sum_{x \in \Xi} p(x)^{q} .
$$

Zebrowski et al. (1994) have defined "pattern entropy" as

$$
Z_{q}(\tau)=-\sum_{x \in \Xi} P_{q}(\tau) \log P_{q}(\tau)
$$

where

$$
P_{q}(\tau)=p\left(x_{1}\right) p\left(x_{2}\right) \ldots p\left(x_{q}\right) .
$$

The variables $X_{i}$ are given as in Eq. (6). The quantity $Z_{q}(\tau)$ is not an entropy in the strict mathematical sense. We will, however, use the term pattern entropy, as it has been introduced by Zebrowski et al. (1994). The product $P_{q}(\tau)$ can be considered as a joint probability distri- bution $p\left(x_{1} ; \ldots ; x_{q}\right)$ only if the variables $X_{1}, \ldots, X_{q}$ are independent. Then $Z q(\tau)=H\left(X_{1} ; \ldots ; X_{q}\right)=H\left(X_{I}\right)+\ldots+$ $H\left(X_{q}\right)$ and for a stationary series $Z_{q}=q H\left(X_{I}\right) \equiv q H^{l}$. For general stationary series $P_{q}=p(x)^{q}$ and

$$
Z_{q}=-\sum_{x \in \Xi} p(x)^{q} \log p(x)^{q},
$$

which does not have usual properties of the entropy: $\mathrm{Zq}$ is the larger the more ordered the analyzed signal is and the additivity properties of the usual entropy are lost. In spite of these peculiarities it has been shown that $\mathrm{Zq}$ is able to distinguish between a high risk and a low risk of cardiac arrest CA (Zebrowski et al., 1994) as well as to estimate other medically interesting properties of heart rate variability (Zebrowski et al., 1999), (Zebrowski et al., 2000). $Z_{q}$ is also a quantity very well correlated with the level of the hormone noreprephanine as measured early in the morning once a day: the correlation coeficient is -0.74 (Poplawska et al., 1994). Pattern entropy was found to correlate with classical quantitative measures of heart rate variability such as SDNN, RMSSD and PNN50 (Zebrowski et al., 1996) indicating that it may be possible to use the pattern entropy to monitor both the sympathetic and the parasympathetic nervous system and avoid the well known problems of the sensitivity of the classical measures to extrasystoles and artifacts.

\section{ESTIMATING ENTROPIES FROM EXPERI- MENTAL DATA}

Estimating entropies and other functionals of probability functions from continuous variables is always problematic and none of various approaches is generally accepted as the best one. Here we will consider the simplest "box-counting" approach, when the probability distribution functions $p\left(x_{1} ; \ldots ; x_{n}\right)$ are estimated as timeaveraged histograms. A state-space partition $\Xi_{1} \times \ldots \times \Xi_{n}$ must be defined and the probability distribution function $p\left(x_{1} ; \ldots ; x_{n}\right)$ is estimated as the relative frequencies of occurrence of time-series samples in particular bins. Consider first the one-dimensional case and probability mass function $p(x)=\operatorname{Pr}\{X=x\}, x \in \Xi$. The elements of $\Xi$, called bins, must be defined on a part of a straight line of length $\mathrm{R}$, given by the extent of the data studied, $\mathrm{R}$ = maximum - minimum, found in the data. The simplest way to define $\Xi$ is using bins of the same width, called equidistant partition. The equidistant partitions were used here for estimations of all of the static entropies. There are still two ways how to define an equidistant partition: Given a number $Q$ of bins, the bin width $\mathrm{W}$ is defined as $W=R=Q$; or, given the bin width $\mathrm{W}$, the number of bins is defined as $Q=R=W$. These approaches are not equivalent, if several time series with different extents $R$ are evaluated. In multidimensional cases, i.e., when estimating entropies or redundancies of 
$n$ variables (which may be defined by time-lagging one variable), the n-dimensional partition $\Xi_{1} \times \ldots \times \Xi_{n}$ will be defined by defining each marginal partition $\Xi_{i}$. Here we will consider using the same $Q(W)$ for each $\Xi_{i}$.

Estimating entropies and redundancies so that the number $Q$ of bins is given and the bin width $W$ is derived according to the range $\mathrm{R}$ of the data (henceforth referred to as "fixed-Q" approach) is desirable, considering the critical effect of Q on the entropy/redundancy estimates. The naive approach to estimate entropies/ redundancies of continuous variables should be the use of the finest possible partition given, e.g., by an available space in computer memory or by measurement precision. We must remember, however, that we usually have a finite number $\mathrm{N}$ of data samples. Hence when using a too fine partition the estimation of entropies and redundancies can be heavily biased: Estimating the joint entropy of $n$ variables using $Q$ marginal bins one obtains $Q^{n}$ boxes covering the state space. If $Q^{n}$ approaches the number $N$ of data samples, or even $Q>N$, the estimate of $H\left(X_{l}\right.$; $\left.\ldots ; X_{n}\right)$ can be equal to $\log (N)$, or, in any case, it can be determined mainly by the number of the data samples and/or by the number of the distinct data values and not by the data structure, i.e. by the dynamical properties of the system under study. Thus even "natural" partitions of experimental data given by an $A / D$ converter is usually too fine for a reliable estimation of entropies and redundancies.

Having a large number $N$ of data samples, the estimated entropy increases with $Q$ as $\log Q$. Thus, classifying time series according to their entropies, using fixed $Q$ is necessary to obtain consistent results. In some cases, however, the fixed-Q approach can have some disadvantages. For instance, consider a set of time series having their basic ranges more or less constant, however, some of the series contain a few outliers, i.e., values far outside the basic range. Then using $W=R / Q$ with a fixed $Q$ can lead to an underestimation of the actual entropy of the series, considering $0 \log 0=0$.

Having defined the bin width $W$ and deriving the number of bins as $Q=R / W$ (henceforth referred to as "fixed-W" approach) can help in the above case with the outliers. Ranges of the time series studied, however, should be approximately constant or an increased variance should be equivalent to "richer dynamics", i.e., to an actual increase of entropy. Otherwise, two series would be classified as having different entropies, though one of them is just the other series multiplied by a constant. In their original work, Zebrowski et al. (1994) have applied the fixed-W approach, considering the argument that in some cases such a linear rescaling of the data series may be of importance (i.e., we would have then RR interval series with a different variance) and the fact that entropy is larger for one of them may be used as an indication of the increase of the variance. This is why - in the case of the pattern entropy where the fixed-W approach is used - the physical interpretation of the changes of the value of this complexity measure are somewhat ambiguous. They may be caused both by an increase of the ordering of the series analyzed (e.g. a drop of the number of frequencies present in the signal) and by a change in variance. So in spite of the fact that the pattern entropy appears to be good at yielding a statistical differentiation between high risk and low risk CA cases, other complexity measures are sought (Zebrowski et al., 1995c).

An approach, different from the above two, was used to estimate the coarse-grained entropy rates CER's. The CER's were computed from mutual information, which was estimated by a fixed-Q box-counting algorithm, however, marginal partitions were not equidistant, but "equiquantal". This means that the marginal bins were defined in such a way that they contained approximately the same number of samples, while their widths were not constant. I.e., effectively the data were transformed into a uniform distribution with marginal entropy $\mathrm{H} 1=$ $\log \mathrm{Q}$ and all dynamical information were obtained from the joint probability functions/histograms. This approach eliminates the effects of outliers. For more details see Ref. (Palus, 1995), (Palus, 1996), (Palus \& Vejmelka, 2007) and references therein.

\section{NUMERICAL DETAILS}

The 24-hour time series of RR intervals are nonstationary, while the entropy measures are defined for stationary processes. Therefore in practical processing we do not estimate the entropy measures from the whole records, but from a short window, which "slides" through the time series in an overlapping way. It is believed, that the violation of the stationarity condition inside a window is not so critical as compared to the whole recording. Thus, in choosing the window length, one needs to compromise between the basic need to use a long enough part of the data series to obtain mathematically meaningful results and the necessity to make this series as short as possible to be able to analyze nonstationary states. Initially, the approach adopted for pattern entropy calculations by Zebrowski et al. (1994, 1995a) was to use stretches of time such as a doctor analyzing an ECG would use. It turned out that usually 5 min. epochs of ECG are used for power spectral density analysis and that a doctor analyzes an ECG visually from recordings of lengths from $30 \mathrm{~s}$ to $5 \mathrm{~min}$. However, using real time creates ambiguities as heart variability changes within a single recording greatly so that the number of data points in a time epoch of predefined length is far from constant. Instead of a real time window, a window with a fixed number of evolutions was adopted. In the initial study a window of 400 beats was used (the equivalent of 3-7 min. of real time) and found to give good statistical predictions on the risk of CA. It has also been shown (Zebrowski et al., 1995b) that a 
statistically reliable result in distinguishing between low risk and high risk groups of CA is obtained for $Z_{q}$ when an apparently too short time window is used (e.g. 50 heart beats or about 30-50 s). For the fixed bin width approach this means that some of the bins of the histogram for a given window may be empty or contain only very few data points. For an estimate of a probability distribution this seems to be inadequate. However, when the window was slid along the data series with a step of every single beat (Zebrowski et al., 1994), it was found that the pattern entropy as a function of time is a reasonably smooth curve. This result indicates that, although some of the information is lost when static entropies are calculated within a single window, sliding the window every single evolution of the system helps alleviate this problem. The mean, maximum and minimum of pattern entropy calculated in such a way allows distinguishing between a low and a high risk of CA both for 24-hour Holter ECG (Zebrowski et al., 1994), (Zebrowski et al., 1995b) and for short Holter (14 min.) ECG recordings (Baranowski et al., 1994). In this paper, in order to obtain more stable results when different complexity measures were calculated, a relatively long time window of 512 beats (6-9 min. of real time) was used throughout. To make the calculations somewhat faster the window was slid every 200 beats and the 24hour histograms made from the resultant entropy values. For the fixed-Q approach the number of bins $Q=16$ was used, while for the fixed-W the bin width $\mathrm{W}=25$ was applied. Wherever applicable the time lag was 2 beats.
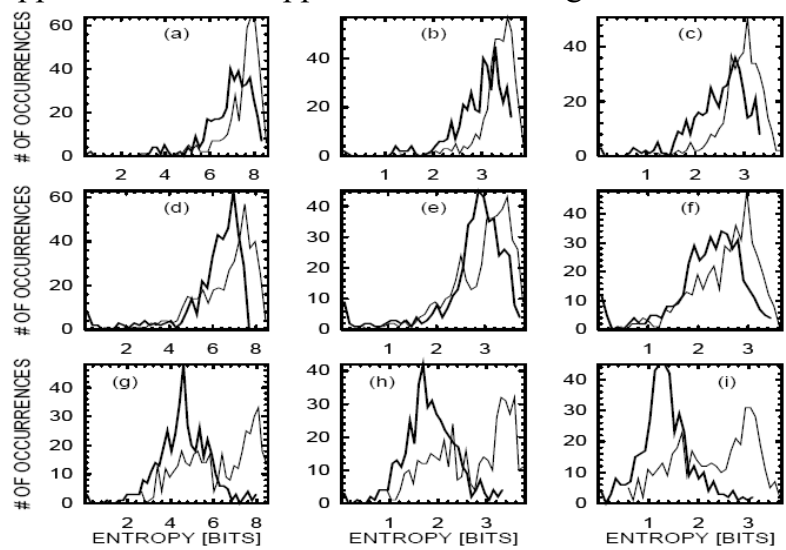

Figure 4. Histograms of the fixed-Q static entropies: the Shannon entropy $H^{l}(\mathrm{~b}, \mathrm{e}, \mathrm{h})$ and the Renyi entropy $H^{3}$ (c, f, i); for the pairs dp1 (a, b, c), dp2 (d, e, f) and dp3 (g, h, i). The histograms of the "a" files and the "b"-files are plotted using the thick and thin lines, respectively.

Note that for a 512 window the time lag of 2 beats is almost negligible. However, this may not necessarily be the case when a short window of 50 or even 100 beats is used, especially when the heart rate variability is extremely low as in deep pathology or using animal data. The coarse-grained entropy rates CER's were computed from n-dimensional probability distributions (histo- grams), $n=2 ; \ldots .$. Therefore the problem of the number of data samples and the number $\mathrm{Q}$ of marginal bins is more critical than in estimations of the static 1dimensional entropies. As a preliminary result we present here the CER's obtained using the window length equal to 4096 beats (an equivalent of $35 \mathrm{~min}$. to 70 min. of real time) and $Q=4, n=2$. The CER $h^{(0)}$ was computed using $\tau_{0}=0 \tau_{1}=1$, for the CER $h^{(l)}$ the lags $\tau_{0}=0$ and $\tau_{\max }=35$ beats were applied. The window step was also 200 beats and the obtained values were processed into histograms of the CER's values in the same way as the static entropies.

\section{RESULTS}

The results for the Shannon and Renyi entropies using fixed-Q partitions are presented in Figure 4, the first row displays the histograms for the pair $\mathrm{dp} 1$, the second for $\mathrm{dp} 2$, the third for $\mathrm{dp} 3$. The histograms for a-files are plotted using thick lines, thin lines are used for b-files. The first column in Figure 4 contains histograms of 3dimensional Shannon entropies $H^{3}(2)$, the second column contains histograms of 1-dimensional Shannon entropies H1, while the histograms of the third-order Renyi entropies H3 are displayed in the third column. Also the second-order Renyi entropies $H^{2}$ were computed, but their histograms were the same as related histograms of $H^{3}$.
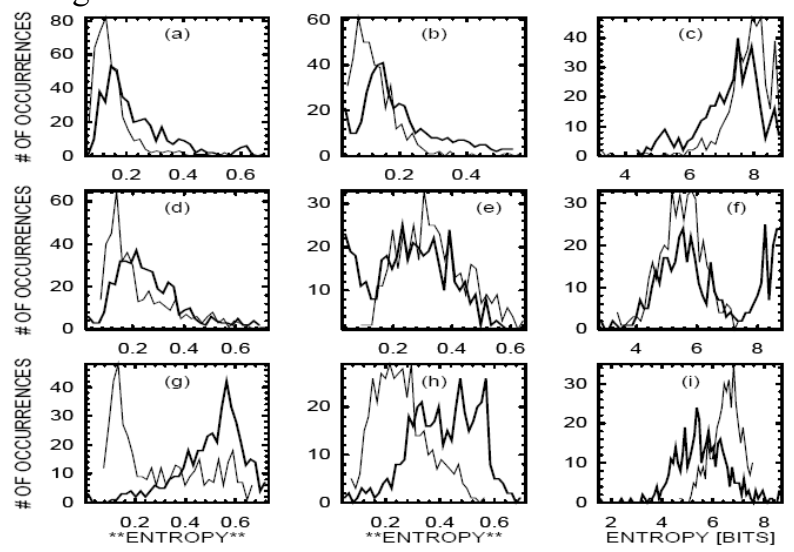

Figure 5. Histograms of the static entropies: the fixed-Q pattern entropy $Z_{3}(2)(\mathrm{a}, \mathrm{d}, \mathrm{g})$, the fixed-W pattern entropy $Z_{3}(2)$ $(\mathrm{b}, \mathrm{e}, \mathrm{h})$, and the fixed-W Shannon entropy $H^{3}(2)(\mathrm{c}, \mathrm{f}, \mathrm{i})$; for the pairs dp1 (a,b,c), dp2 (d,e,f) and dp3 (g,h,i). The histograms of the "a"-files and the "b"-files are plotted using the thick and thin lines, respectively.

The results of all entropies in Figure 4 are consistent histograms of a-files (thick lines) are located on the leftsides of the histograms of the b-files. Thus the Shannon and Renyi entropies estimated using the fixed-Q approach sort all the a-files into one group and all the $b$ les into the other group. The same sorting is obtained from the 3 rd order pattern entropy $Z^{3}(2)$, if it was also estimated using fixed-Q partitions (first column in Figure 5). When the 3 -rd order pattern entropy $Z^{3}(2)$ is es- 
timated using the fixed-W partition (the second column in Figure 5), the results for $\mathrm{dp} 1$ and $\mathrm{dp} 3$ are more or less consistent with the fixed-Q approach, however, this is not the case for $\mathrm{dp} 2$. It seems that now the sorting is a$b-a$ and $b-a-b$, unlike the $a-a-a$ and $b-b-b$ sorting found above. This result is not typical for the pattern entropy, but can be obtained from other static entropies, when fixed-W partitions are used. An example for the fixedW Shannon entropy $H^{3}(2)$ is presented in the third column of Figure 5.

Considering the original health-pathology (patientcontrol) coding, does this mean, that the fixed-W partition is appropriate for detection of pathology in this case? We will see that this example is not appropriate to decide this problem. First, however, we report results from dynamical entropies - coarse grained entropy rates CER. CER $h^{(1)}$ - the first column in Figure 6, CER $h^{(0)}$ the second column in Figure 6. At the first sight it seems, that the sorting given by CER's is even worse than that given by the static entropies, and giving a fixed-Q-like (i.e. incorrect) sorting. There is one feature, however, in the CER's histograms, which in this case provide the correct $a-b-a / b-a-b$ sorting. It is the existence of small values $\left(0.6-0.8\right.$ in CER $h^{(1)}$, and 0.4 - 0.6 in CER $h^{(0)}$ ), which occurs only in the case of the controls.
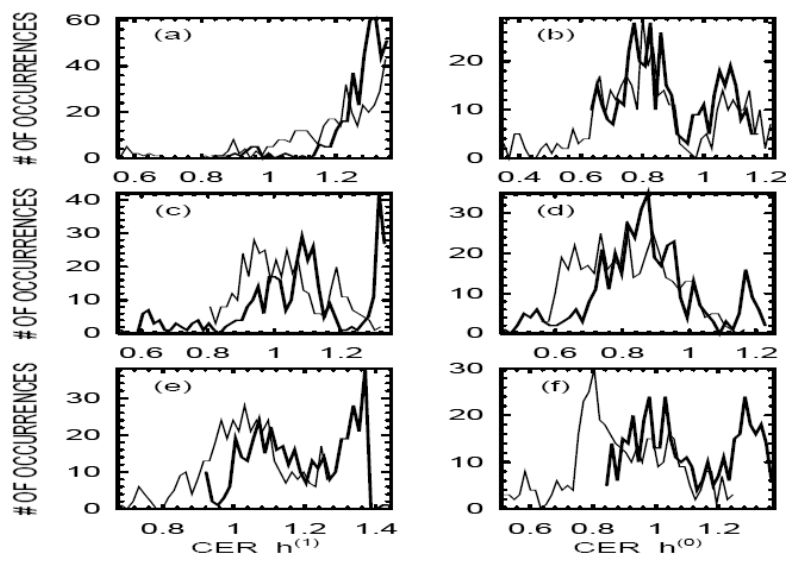

Figure 6. Histograms of the dynamic entropies - the coarsegrained entropy rates CER $h^{(l)}(\mathrm{a}, \mathrm{c}, \mathrm{e})$ and CER $h^{(0)}(\mathrm{b}, \mathrm{d}, \mathrm{f})$; for the pairs dp1 $(a, b), d p 2(c, d)$ and dp3 $(e, f)$. The histograms of the "a"-files and the "b"-files are plotted using the thick and thin lines, respectively

Let us concentrate on the dp2 pair and results which discriminate the a-file here correctly as the control. In the case of the fixed-W static entropies, the results can be influenced just by different variance of the data. And this is the case of dp2a (Figure 2a), where the last third of the data has much larger variance than the rest of the record. Let us rerun the computations for the dp2 pair, but now only for the first 50,000 samples, for the Shanon and pattern entropies (fixed-W) and for the CER's. The results are presented in Figure 7 . The static entro- pies (a - Shannon, c - pattern) lost the part that distinguished a from b, while the low-CER part of the histogram is still present in the histograms of the CERs.

From the point of view of characterizing "complexity of dynamics" it seems that the dp2a-dp2b distinction by the static entropies was obtained due to an artifact - the larger variance seen in the last third of Figure 2a. From the point of view of practical medical diagnostics, however, the variance itself can be an important factor. The fixed-W static entropies are sensitive both to a larger variance and to a possibly accompanying change in the level of ordering in the signal. The first 50,000 points in the series represent heart rate variability during the day. It is well known that there are people whose heart rate variability during the day may be very different from that during the night. Often dangerous symptoms occur only during the night and not during the activity periods (or vice versa). In the case of the patient $d p 2 b$, the relatively uniform variance throughout the 24 hours was a danger signal for his doctors. On the other hand, the large increase of variance in the control dp2a in the night hours was considered normal. In general, however, the value of the variance of the RR intervals itself is not a suffcient indicator of a risk of impending cardiac arrest. The fixed-W pattern entropy successfully classified the target member $d p 2 b$ to be in the high risk class he died due to CA several months afterwards. The question of discriminating power of dynamics versus variance, however, still needs further investigation.
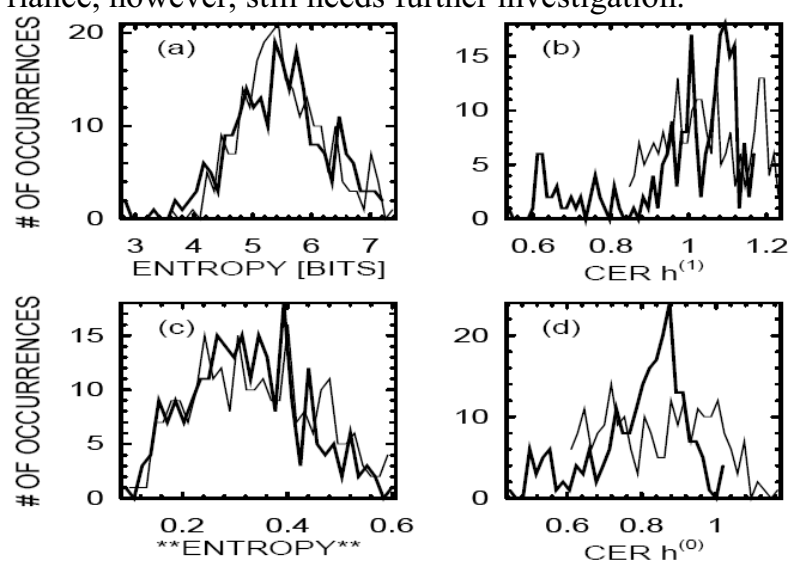

Figure 7. Histograms of entropies and entropy rates for the pair dp2, computed from the first 50,000 samples (i.e., excluding the high variance part of dpla, see Figure 2), the fixed-W Shannon entropy $H^{3}(2)(a)$, the fixed-W pattern entropy $Z_{3}(2)$ (c), CER $h^{(l)}(b)$ and CER $h^{(0)}(d)$. The histograms of the "a"files and the "b"-files are plotted using the thick and thin lines, respectively.

The interesting finding here is that the difference between the members of the pair 2, as detected by the CER's, persists in the data in general, not only in the visibly high variance part of the data. Thus it is possible, that the distinction between health and pathology may be better detectable in the complexity of dynamics 
as measured by (coarse-grained) entropy rates. In other words, there are features of the RR series detected by the CER's, which cannot be found in the static complexity of the marginal distributions. This result, however, is very preliminary, and a much larger database of RR recordings need to be processed to confirm validity of this finding.

\section{Acknowledgement}

The authors thank Dr. Wanda Poplawska for very important discussions and for selecting and providing the RR-interval data. Milan Palus was supported by the Institutional Research Plan AV0Z10300504.

\section{REFERENCES}

Baranowski, R., Zebrowski, J.J., Poplawska, W., \& Rywik, S. (1994). Nonlinear dynamical methods in heart rate variability analysis from short Holter recordings. Journal of Ambulatory Monitoring, 7, 81 .

Cover, T.M., \& Thomas, J.A. (1991). Elements of Information Theory. New York: Wiley \& Sons.

Fraser, A.M. (1989). Information and entropy in strange attractors. IEEE Transactions on Information Theory, 35, 245262.

Hlavackova-Schindler, K., Palus, M., Vejmelka, M., \& Bhattacharya, J. (2007). Causality detection based on informationtheoretic approaches in time seriesanalysis. Physics Reports, 441, 1-46.

Palus, M. (1993). Identifying and quantifying chaos by using information-theoretic functionals. In: A.S. Weigend N.A. \& Gershenfeld (Eds.), Time Series Prediction: Forecasting the Future and Understanding the Past. Santa Fe Institute Studies in the Sciences of Complexity, Proc. Vol. XV, pp. 387-413. Addison-Wesley: Reading, Mass.

Palus, M. (1995). Testing for nonlinearity using redundancies: Quantitative and qualitative aspects. Physica D, 80, 186-205.

Palus, M. (1996). Coarse-grained entropy rates for characterization of complex time series. Physica D, 93, 64-77.

Palus, M., \& Vejmelka, M. (2007). Directionality of coupling from bivariate time series: How to avoid false causalities and missed connections. Physical Review E, 75, 056211.

Petersen, K. (1983). Ergodic Theory. Cambridge: Cambridge University Press.

Poplawska, W., Grzybowski, J., Zebrowski J.J., Baranowski, R., Bilinska Z.T., \& Pszona, B. (1994). Pattern Entropy - nonlinear noninvasive estimation of adrenergic drive in patients with dilated cardiomyopathy. Journal of Ambulatory Monitoring, 7,80 .

Sinai, Y.G. (1976). Introduction to Ergodic Theory. Princeton: Princeton University Press.

Zebrowski, J.J., Poplawska, W., \& Baranowski, R. (1994). Entropy, pattern entropy and related methods for the analysis of data on the time intervals between heart beats from 24 hour electrocardiograms. Physical Review E, 50, 4187-4205.

Zebrowski, J.J., Poplawska, W., \& Baranowski, R. (1995a). Pattern Entropy a Tool for Nonlinear Dynamical Analysis of a Biological Nonstationary System: The Human Heart. Acta Phyica Polonica B, 26, 1055-1064.

Zebrowski, J.J., Poplawska, W., Baranowski, R., \& Buchner, T. (1995b). Local Time Properties of 24- hour Holter recor- dings of RR-intervals: a nonlinear quantitative method through pattern entropy of 3-D return maps. Computers in Cardiology, 377, 177-180.

Zebrowski, J.J., Poplawska, W., Baranowski, R., \& Buchner T. (1995c). Pattern entropy a tool for nonlinear dynamical analysis of a biological nonstationary system: The human heart. Acta Physica Polonica B, 26, 1055-1064.

Zebrowski, J.J., Poplawska, W., Baranowski, R., \& Buchner T. (1996). Nonlinear analysis of heart dynamics. Medical \& Biological Engineering \& Computing, 34 (Supplement 1, Part I), 375-376.

Zebrowski, J.J., Poplawska, W., Baranowski, R., \& Buchner, T. (1999). Measuring the Complexity of Nonstationary Time Series - Nonlinear Interpretations of Selected Physiological Processes. Acta Physica Polonica B, 30, 2547-2570.

Zebrowski, J.J., Poplawska, W., Baranowski, R., \& Buchner, T. (2000). Symbolic dynamics and complexity in a physiological time series Chaos, Solitons and Fractals, 11, 1061-1075. 